Https://online.unisc.br/seer/index.php/cadpesquisa

ISSN on-line: $1677-5600$

Doi: 10.17058/cp.v31i1.12273

Universidade de Santa cruz do Sul - Unisc

Recebido em 24 de Junho de 2018 Aceito em 27 de Março de 2019 Autor para contato: nascimentob.vn@gmail.com

\title{
Herpetofauna do Parque Estadual Ecoturístico e de Desenvolvimento Sustentável da Cachoeira do Urubu, Primavera, Pernambuco, Brasil
}

\author{
Herpetofauna of Parque Estadual Ecoturístico e de Desenvolvimento Sustentável da \\ Cachoeira do Urubu, Primavera, Pernambuco, Brazil
}

Vanessa do Nascimento Barbosa

Universidade Federal Rural de Pernambuco - UFRPE - Recife - Pernambuco - Brasil

Jéssica Monique da Silva Amaral

Programa de Pós Graduação em Ecologia e Monitoramento Ambiental - UFPB - João Pessoa - Paraíba - Brasil

\author{
Katharina Siqueira Nino \\ Luiz Filipe Lira Lima \\ Igor Yuri Gonçalves Silva dos Santos \\ Universidade Federal Rural de Pernambuco - UFRPE - Recife - Pernambuco - Brasil \\ Gleymerson Vieira Lima de Albuquerque \\ Escola Técnica Estadual de Pernambuco - Recife - Pernambuco - Brasil \\ Ednilza Maranhão dos Santos \\ Universidade Federal Rural de Pernambuco - UFRPE - Recife - Pernambuco - Brasil
}

\section{Resumo}

Este trabalho objetivou inventariar a herpetofauna do Parque Estadual Ecoturístico e de Desenvolvimento Sustentável da Cachoeira do Urubu, destacando ocupação dos organismos nos diferentes habitats e microhabitats, bem como seu status de conservação. A coleta dos dados foi realizada nos meses de julho de 2015 e janeiro de 2016, através de buscas ativas, passivas e registros de terceiros. Foram registradas 21 espécies de anfíbios distribuídos em cinco famílias e 18 espécies de répteis distribuídos entre 11 famílias. A lista apresentada neste trabalho vem contribuir com informações sobre a herpetofauna em áreas de Mata Atlântica e como ela está representada em uma área onde existe um nível de antropização relacionado diretamente a atividades turísticas.

\section{Abstract}

This paper main objective is making an inventory of the herpefauna in the "Parque Estadual Ecoturístico e de Desenvolvimento Sustentável da Cachoeira do Urubu", highlighting the occupation of organisms in different habitats and microhabitats, as well as their conservation status. The data collection occurred in July 2015 and in January 2016, through active, passive search and personal relates. Twenty one species of amphibians were registered, distributed in five families, and 18 species of reptiles distributed in 11 families. The presented list in this paper contributes with information about herpetofauna in areas of Atlantic Forest and how it is represented in an area with a level of anthropization which is directly related to touristic activities.

\section{Palavras-chave}

Conservação. Mata Atlântica. Nordeste brasileiro. Centro de endemismo.

\section{Keywords}

Conservation. Atlantic Forest. Northeast Brazil. Endemic center. 


\section{Introdução}

A herpetofauna inclui dois grupos importantes, os anfíbios e os répteis, e ambos representam uma das maiores diversidades de vertebrados terrestres do planeta (Segalla et al., 2016; Costa e Bérnils, 2018). No Brasil, o grupo dos anfíbios se destaca liderando o ranking mundial. Atualmente, a lista de espécies conta com 1026 táxons distribuídos em três ordens: Anura (998 espécies); Urodelos (cinco espécies) e Gimnophionas (22 espécies), compreendendo 24 famílias (Segalla et al., 2016). Esses animais são considerados como ótimos bioindicadores de qualidade do ambiente (Bertoluci et al., 2009; Lopes, 2010), biocontroladores de invertebrados e pequenos vertebrados, além de servir de alimento para outros táxons e atuarem significantemente na bioprospecção (Bertoluci et al., 2009) e, portanto, são considerados sentinelas ambientais.

Em relação aos répteis, no Brasil, esse grupo ocupa a terceira posição em riqueza de espécies do mundo, possuindo 795 espécies que estão divididas entre os clados Testudine (36 espécies), Squamata (753 espécies), das quais se tem como representantes as amphisbaenas (72 espécies), os lagartos (276 espécies) e as serpentes (405 espécies), e Crocodylia (seis espécies) (Costa e Bérnils, 2018). Os répteis são também considerados bioindicadores de qualidade de ambiente, respondendo às variações do meio, à presença de espécies invasoras e à ocorrência de queimadas (Filho, 2003). São também excelentes biocontroladores, principalmente de artrópodes, além de que podem ser excelentes dispersores de sementes (Santos et al., 2012). Alguns desses animais são utilizados como animais de estimação (Muriarty, 2002), na medicina popular e na bioprospecção (Oliveira, 2013; Araújo e Luna, 2017), e como alimento (Alves et al., 2010; Araújo e Luna, 2017).

Apesar da grande riqueza de anfíbios e répteis no Brasil, várias espécies estão ameaçadas graças à destruição dos ecossistemas naturais, principalmente daqueles que são considerados hotspots pra conservação, como a Mata Atlântica (Mello et al., 2016). No estado de Pernambuco este Bioma possui cerca de 60 espécies de anfíbios anuros (Moura et al., 2011ab), dos quais 60\% são endêmicas (Haddad e Prado, 2005; Segalla et al., 2016), e possuem pelo menos 37 modos reprodutivos intimamente dependentes dos ambientes úmidos (Haddad et al., 2013). Em relação aos répteis, a Mata Atlântica comporta cerca de 200 das 795 espécies encontradas no Brasil e, dessas, cerca de metade estão reportadas para a Mata Atlântica de Pernambuco (Moura et al., 2011a).

Quanto à Mata Atlântica nordestina, apesar de bastante fragmentada e infinitamente menor que sua forma original, seus remanescentes pertencentes ao estado de Pernambuco são pouco investigados, principalmente aqueles que estão na Zona da Mata Sul (Roberto et al., 2017), onde atualmente encontra-se o Parque Estadual Ecoturístico e de Desenvolvimento Sustentável da Cachoeira do Urubu (PECUB). O PECUB é um importante complexo de mananciais hídricos, possuindo cachoeiras, ambientes aquáticos lênticos e lóticos permanentes, além de áreas encharcadas, como poças temporárias, nos quais servem de micro e macro ambientes propícios à presença da herpetofauna. O objetivo deste trabalho é inventariar a fauna de anfíbios e répteis do PECUB, buscando somar e divulgar informações que fomentem a conservação local, bem como contribuir com o plano de manejo e zoneamento da área. 


\section{Material e métodos}

\section{Área de estudo}

Na mata sul do estado de Pernambuco encontra-se o Parque Estadual Ecoturístico e de Desenvolvimento Sustentável da Cachoeira do Urubu (PECUB), localizado na cidade de Primareva, distante $81,6 \mathrm{~km}$ de Recife, capital de Pernambuco (Fig. 1). No Estado de Pernambuco, a Mata Atlântica sul é classificada como floresta estacional semi-decidual submontana e, por desenvolver-se em solo mais seco e possuir dupla estacionalidade climática, tem períodos secos e chuvosos em equilíbrio, o que mantém um balanço hídrico importante em sua vegetação (Uchoa-Neto e Tabarelli, 2002).

Figura 1: Localização geográfica do Parque Ecoturístico de Desenvolvimento Sustentável da Cachoeira do Urubu, localizada no estado de Pernambuco Nordeste do Brasil.

O PECUB possui área de $300.000 \mathrm{~m}^{2}$ (30 hectares), dos quais $80 \%$ é uma reserva de proteção integral e $20 \%$ de uso sustentável, e possui uma queda d'água de $77 \mathrm{~m}$ de altura. A cidade de Primavera faz fronteira, ao Norte, com os municípios de Pombos e Chã Grande; ao Sul, faz fronteira com o município de Ribeirão; à Leste, faz divisa com os municípios de Escada e Vitória de Santo Antão; e à Oeste, limita-se com o município de Amaraji. A área é acessada pela rodovia federal BR-101 e pela rodovia estadual PE-063. O parque atualmente recebe um variado público e tem em seu espaço bares e lanchonetes, assim como residências fixas de moradores que vivem no local desde a sua formação. $O$ trabalho analisou três diferentes áreas do PECUB sendo caracterizados quanto às suas paisagens (Tab. 1; Fig. 2).

Tabela 1. Áreas amostradas e ambientes ocupados pela herpetofauna no Parque Estadual Ecoturístico e de Desenvolvimento Sustentável da Cachoeira do Urubu durante o período de 20 a 24 de julho de 2015 e 04 a 08 de janeiro de 2016, com informações georreferenciadas.

Figura 2: Imagens dos ambientes amostrados para herpetofauna do Parque Estadual Ecoturístico e de Desenvolvimento Sustentável da Cachoeira do Urubu durante o período de 20 a 24 de julho de 2015 e 04 a 08 de janeiro de 2016. (1) Capinzal, (2) Queda d'água, (3) Braço do rio, (4) Bananal, (5) Compostagem, (6) Bica, (7) Piscina, (8) Bares.

Procedimento metodológico

As coletas ocorreram em duas campanhas, uma na estação chuvosa, entre os dias 20 e 24 de julho de 2015, e outra na estação seca, entre os dias 04 e 08 de janeiro de 2016. 0 esforço foi dividido em coletas diurnas (08:00 $\mathrm{h}$ às 16:00 h) e noturnas (18:00 h às 23:00), através de procura visual limitada por tempo (PVLT), com observação direta e indireta (zoofonia), vestígios, registro de terceiros e armadilha de corvo (Fig. 3), esta última foi alocada e presa nas margens do rio utilizando como iscas vísceras de frango.

Os espécimes foram identificados usando chaves artificiais para cada grupo com base na folidose (Peters e Orejas-Miranda, 1970; Peters e Barros, 1970; Bernarde, 2014), medidos com paquímetro digital (precisão de 0,01 $\mathrm{mm}$ ) e trena e pesados com balança digital (precisão de $0,1 \mathrm{~g}$ ) e pesola Ligth-Line (precisão de $0,3 \%$ carga). Posteriormente foi feita a identificação do 
sexo com equipamento apropriado (sexador) e soltos nos locais de captura, todavia, alguns exemplares foram eutanasiados através de superdosagem de Ketamina, fixados em formalina $10 \%$ e conservados em álcool a $70 \%$. Posteriormente depositados para o tombamento na Coleção Herpetológica e Paleoherpetológica da Universidade Federal Rural de Pernambuco (CHP-UFRPE: 5049-5091; licença do SISBIO 11218-1).

Figura 3: Métodos utilizados para o inventário rápido no Parque Estadual Ecoturístico e de Desenvolvimento Sustentável da Cachoeira do Urubu durante o período de 20 a 24 de julho de 2015 e 04 a 08 de janeiro de 2016. A- Método de procura visual limitada por tempo; BMontagem da armadilha tipo corvo; C- Armadilha tipo corvo no braço do rio.

Para análise numérica dos resultados, foram realizados cálculos de constância de ocorrência de cada espécie de acordo com o método proposto por Dajoz (1983), cujos dados percentuais são obtidos a partir da equação $C=p * 100 / P$, na qual: $C=$ constância de ocorrência de cada espécie, $\mathrm{p}=$ número de excursões em que a espécie foi registrada e $\mathrm{P}$ = número total de excursões. Tal análise define os espécimes em três categorias: constantes (se ocorreram em mais de $50 \%$ da amostra), acessórios (se ocorreram entre $25 \%$ e $50 \%$ das amostras) ou acidentais (se ocorreram em menos de $25 \%$ da amostra).

\section{Resultados e discussões}

Foi registrado um total de 39 espécies com maior representação dos anfíbios, que corresponde a 54\% do total registrado (Tabela 2). Todos os 21 anfíbios registrados são da ordem Anura, distribuídos entre cinco famílias, com maior representatividade para Hylidae, com relação aos répteis, registramos 18 espécies distribuías em 11 famílias, com maior representatividade para a Dipsadidae.

Tabela 2: Herpetofauna Parque Ecoturístico de Desenvolvimento Sustentável da Cachoeira do Urubu, durante o período de 20 a 24 de julho de 2015 e 04 a 08 de janeiro de 2016. Com informações sobre: Local de registro. A1 - Área aberta com predominância de poças e quedas d'água, subdividida em: a- Área do container, b- Queda d'água, c- Braço do rio. A2 - Área florestada com abundância de plantas exóticas, subdividida em: d- Plantação de bananeiras, e- Área de compostagem, f-Mata que percorre a nascente para a bica. A3 - Área edificada: g- bares e piscinas (artificiais). Tipo de registro: busca ativa, busca passiva e relato de terceiros. Frequência de ocorrência: Acidental - Ac; Acessória - As; Constante - Ct. Abundância - número de indivíduos • Espécies que tiveram indivíduos tombados.

O local com maior abundância de anfíbios (Figura 4) foi a área 2 por ser a área mais florestada e com corpos d'água permanentes, seguida da área 3, onde há piscinas (corpos d'água artificiais), tendo um maior número de indivíduos do gênero Rhinella. Em seguida, a área 1 (mais aberta), onde a maioria dos anfíbios foram registrados próximos à queda d'água da cachoeira e na margem do braço do rio. Algumas espécies generalistas foram registradas próximas às edificações, como a perereca de banheiro (Scinax $x$-signatus), tendo em vista que 
espécies com maior plasticidade ambiental se beneficiam com a criação artificial de áreas para reprodução (Grandinetti e Jacobi, 2005).

Figura 4: Distribuição das espécies de anfíbios nas áreas do Parque Estadual Ecoturístico e de Desenvolvimento Sustentável da Cachoeira do Urubu durante o período de 20 a 24 de julho de 2015 e 04 a 08 de janeiro de 2016.

Com relação aos répteis, a área 3 teve o maior registro de indivíduos, corroborando com os relatos dos moradores, seguida da área 1 , onde algumas espécies como o Tropidurus semitaeniatus e Phrynops geoffroanus só tiveram registro nesta área, associada a afloramentos rochosos e à presença de corpos d'água respectivamente, concordando com os hábitos destes animais. Por último, houve registro de apenas cinco espécies na área 2 (Fig. 5).

Figura 5: Distribuição das espécies de répteis nas áreas do Parque Estadual Ecoturístico e de Desenvolvimento Sustentável da Cachoeira do Urubu durante o período de 20 a 24 de julho de 2015 e 04 a 08 de janeiro de 2016.

As espécies Hypsiboas albomarginata e Phyllodytes luteolus foram registradas em todas as áreas, essa última estando associada à presença de bromélias e sendo considerada como especialista de hábitat (Motta-Tavares et al., 2016). Quanto aos répteis, Tropidurus hispidus e Hemidactylus mabouia ocuparam mais ambientes, por serem considerados mais generalistas de hábitat e mais adaptadas a mudanças ambientais, conseguindo se destacar em áreas antropizadas (Bertoluci et al., 2009; Santana et al., 2014).

De uma maneira geral, a maioria das espécies registradas para o PECUB possui ampla distribuição geográfica e são generalistas de hábitat (Santana et al., 2008; Souza et al., 2013; Guedes et al., 2014; Caldas et al., 2016). A presença de espécies que se beneficiam da ação antrópica como Rhinella jimi, Scinax x-sgnatus, T. hispidus e H. mabouia aponta para uma área impactada e que necessita de uma ação de recuperação.

Ao estudar a Zona da Mata Sul de Pernambuco, Lima (1998), verificou que $48 \%$ dos fragmentos são menores que 10 ha e somente $7 \%$ deles são maiores que 100 ha devido à cultura da cana-de-açúcar, que foi a principal causa do desmatamento no estado, ameaçando a permanência das espécies, principalmente de anfíbios e répteis, que são sensíveis tanto à perda de hábitat quanto à ampliação do efeito de borda para o interior dos fragmentos (Moura et al., 2011b).

Dentre as áreas mais relevantes deste ecossistema, encontra-se o Centro de Endemismo de Pernambuco, o qual envolve os remanescentes de Mata Atlântica que ocorrem entre os estados de Alagoas ao Rio Grande do Norte (ao norte do Rio São Francisco) (Santos, 2006). Suspeitava-se que um terço da sua vegetação estaria em risco de extinção devido a uma deficiência no processo de dispersão de sementes, mas estudos de modelagem apontaram que a situação poderia ser mais crítica e que a floresta ao norte do Rio São Francisco é a unidade biogeográfica de Mata Atlântica com a maior vulnerabilidade de perder espécies em escala regional e global (Tabarelli et al., 2005).

Apesar do presente trabalho ser um inventário rápido, houve um esforço maior de utilização do método de buscas ativas, pois é o mais comum em alguns ambientes nos inventários de anfíbios e répteis, tendo em vista que, para alguns táxons, ele tende a 
subamostrar, como no caso de animais especialistas de folhedo, de dossel e aqueles que são fossoriais (Bernarde, 2012). Um estudo a longo prazo proporcionaria o registro de alguns táxons que são sensíveis às questões sazonais (Pereira et al., 2016), e como isso acredita-se que mais espécies poderiam ser acrescidas à presente lista. Mesmo com um esforço de 10 dias em campo, foi possível documentar uma expressiva diversidade de espécies correspondente a 23,75\% da herpetofauna para o estado de Pernambuco, 6,33\% para a Mata Atlântica, e 2,11\% para a lista brasileira de anfíbios e répteis (Rodrigues, 2005; Moura et al., 2011ab; Segalla et al., 2016; Costa e Bérnils, 2018).

Quanto aos métodos de amostragem, houve um total de 32 espécies encontradas através da busca ativa, seis espécies registradas através de relatos de terceiros (moradores e funcionários do PECUB) e apenas uma encontrada através da busca passiva. É necessário levarse em consideração que, para busca passiva, foram usadas apenas armadilhas do tipo covo, na qual se aplica somente a animais aquáticos.

As informações vindas de terceiros foi um bom modo de apontar questões de uso de alguns animais como alimento, como é o caso do teiu/tejú (Salvator meriane) e da gia pimenta (Leptodactylus vastus), caçado por alguns moradores da região. Alves e colaboradores (2010) também relataram o uso de répteis como alimento de caça para os humanos não apenas em zonas rurais como também em centros urbanos.

Não houve registro de espécies endêmicas no PECUB. Algumas espécies citadas neste trabalho já foram registradas em outros estudos para a mata sul de Pernambuco (e.g. Santos e Santos, 2011; Roberto et al., 2017). Entretanto, dez espécies foram registradas pela primeira vez na mata sul do estado de Pernambuco, sendo elas dois anuros, Phyllodytes luteolus e Adenomera marmorata; um testudine, Phrynops geoffroanus; um lagarto, Hemidactylus mabouia, que, mesmo sendo uma espécie exótica, sobrevive bem no Brasil sem causar danos e é inclusa na lista de répteis brasileiros (Costa e Bérnils, 2018); e seis das onze espécies de serpentes registradas, Epicrates cenchria, Chironius carinatus, Chironius flavolineatus, Clelia plumbea, Erythrolamprus viridis e Micrurus ibiboboca.

A serpente surucucu-pico-de-jaca (Lachesis muta) foi registrada no Parque Estadual Ecoturístico e de Desenvolvimento Sustentável da Cachoeira do Urubu apenas através de relato de terceiros, mas foi uma das espécies mais abundantes da Serra do Urubu (Roberto et al., 2017). Essa serpente é o maior representante da família Viperidae, podendo chegar a 3,5m de comprimento (Melgarejo, 2013) e tem risco de entrar na lista de animais ameaçados de extinção por habitar áreas fragmentadas da Mata Atlântica (Alves et al., 2014).

\section{Considerações finais}

Por se tratar de um parque ecoturístico de grande interferência humana, deve-se focar em esforços para a conservação do PECUB, pois nossos resultados apontam para a presença considerável de espécies que ou se beneficiam da presença humana, ou que são especialistas de hábitat, que são as mais sensíveis às alterações do ambiente. A Mata Atlântica, de modo geral, vem sendo muito explorada e fragmentada, e como o PECUB é muito apreciado pela sua paisagem, a população deve se sentir pertencente à área e se tornar agente de conservação. 


\section{Figura 1}

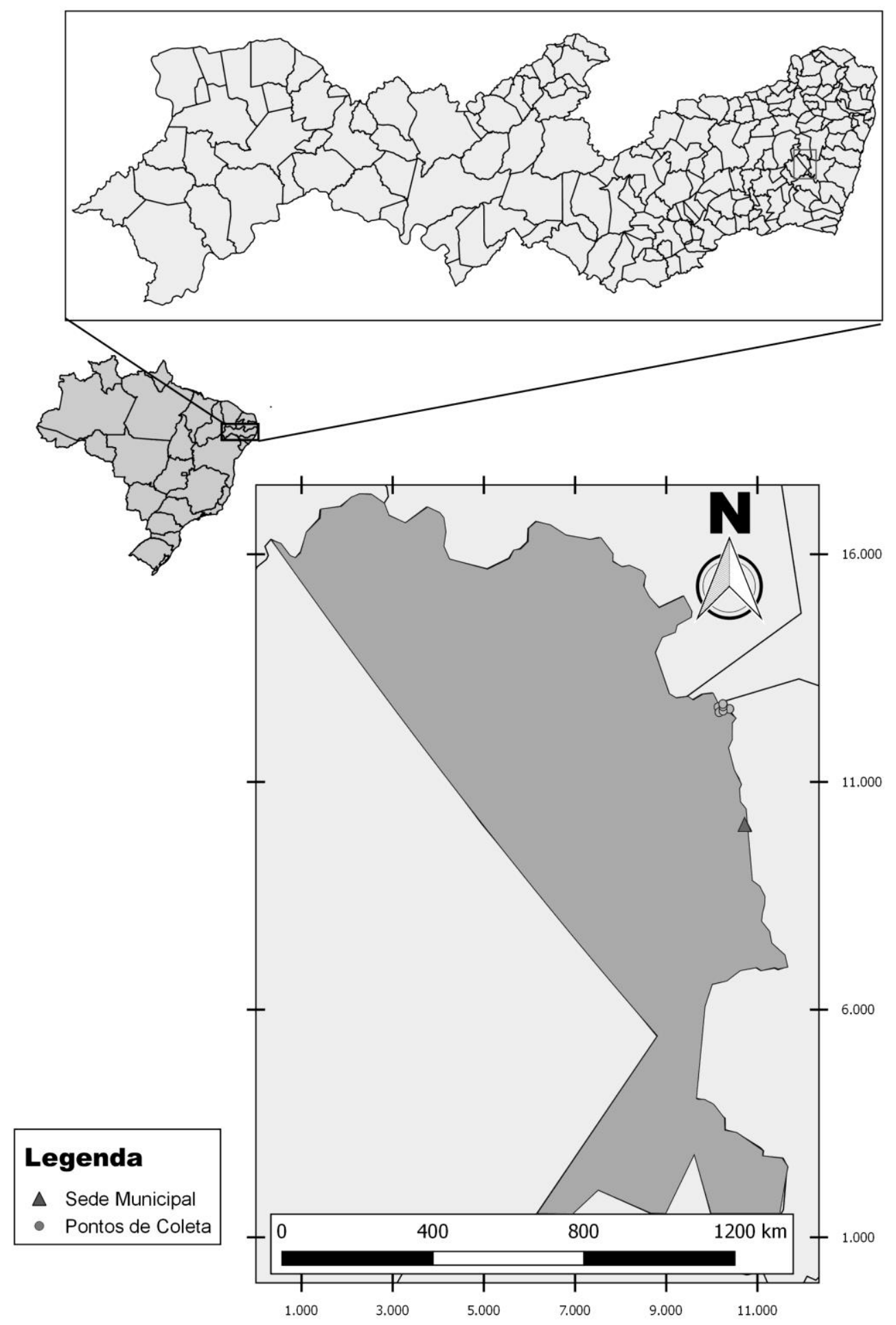

Caderno de Pesquisa [ISSN 1677-5600]. Santa Cruz do Sul, v. 31, n. 1, p. 31-45, jan./abr. 2019 https://online.unisc.br/seer/index.php/cadpesquisa/index 
Tabela 1

\begin{tabular}{|c|c|c|c|c|c|}
\hline \multicolumn{2}{|c|}{$\begin{array}{l}\text { Características } \\
\text { das áreas }\end{array}$} & \multirow{2}{*}{$\begin{array}{c}\text { Ambientes } \\
\text { Capinzal }\end{array}$} & \multirow{2}{*}{$\begin{array}{l}\text { Sigla } \\
C Z\end{array}$} & \multirow{2}{*}{$\begin{array}{c}\text { Coordenada } \\
0240229 \\
9081632\end{array}$} & \multirow{2}{*}{$\begin{array}{c}\text { Caracterização dos } \\
\text { ambientes }\end{array}$} \\
\hline Área 1 & Aberta & & & & \\
\hline & & Queda d'água & QD & & $\begin{array}{l}\text { Queda d'água natural } \\
\text { permanente, com intenso }\end{array}$ \\
\hline & & & & 9081717 & $\begin{array}{l}\text { fluxo de água. No seu } \\
\text { entorno a presença de } \\
\text { afloramentos rochosos, com } \\
\text { vegetação arbustiva e } \\
\text { arbórea próxima a } \\
\text { cachoeira. }\end{array}$ \\
\hline & & Braço do rio & $B R$ & $\begin{array}{l}0240259 \\
9081506\end{array}$ & $\begin{array}{l}\text { Corpo d'água natural e } \\
\text { permanente, com fluxo de } \\
\text { água lótico e lênticos, onde } \\
\text { se pode encontrar plantas } \\
\text { aquáticas flutuantes e } \\
\text { afloramento rochoso. }\end{array}$ \\
\hline \multirow[t]{3}{*}{ Área 2} & $\begin{array}{c}\text { Floresta } \\
\text { da }\end{array}$ & Bananeira & BM & $\begin{array}{l}0240259 \\
9081506\end{array}$ & $\begin{array}{l}\text { Presença de bananeiras, } \\
\text { solo rico em serrapilheira e } \\
\text { bastante úmido. Presença } \\
\text { de um córrego. }\end{array}$ \\
\hline & & Compostagem & $\mathrm{CP}$ & $\begin{array}{l}0240513 \\
9081592\end{array}$ & $\begin{array}{c}\text { Uma parte de área aberta } \\
\text { constituída por capinzal, e } \\
\text { árvores frutíferas, com } \\
\text { predominância de jaqueira e } \\
\text { mangueira. }\end{array}$ \\
\hline & & Bica & $\mathrm{MB}$ & $\begin{array}{l}0240345 \\
9081552\end{array}$ & $\begin{array}{c}\text { Queda d'água artificial e } \\
\text { permanente onde há a } \\
\text { formação de uma pequena } \\
\text { massa de água é circundada } \\
\text { pela vegetação arbórea, } \\
\text { com predominância de } \\
\text { sombreiro. }\end{array}$ \\
\hline \multirow[t]{2}{*}{ Área 3} & $\begin{array}{c}\text { Edificad } \\
\quad a\end{array}$ & Piscinas & OS & $\begin{array}{l}0240356 \\
9081654\end{array}$ & $\begin{array}{l}\text { Construções de pequenas } \\
\text { piscinas edificadas para } \\
\text { recreação dos visitantes. }\end{array}$ \\
\hline & & Bares & BA & $\begin{array}{l}0240337 \\
9081607 \\
\end{array}$ & $\begin{array}{l}\text { Casas e bares construídos } \\
\text { em torno das piscinas. }\end{array}$ \\
\hline
\end{tabular}




\section{Figura 2}
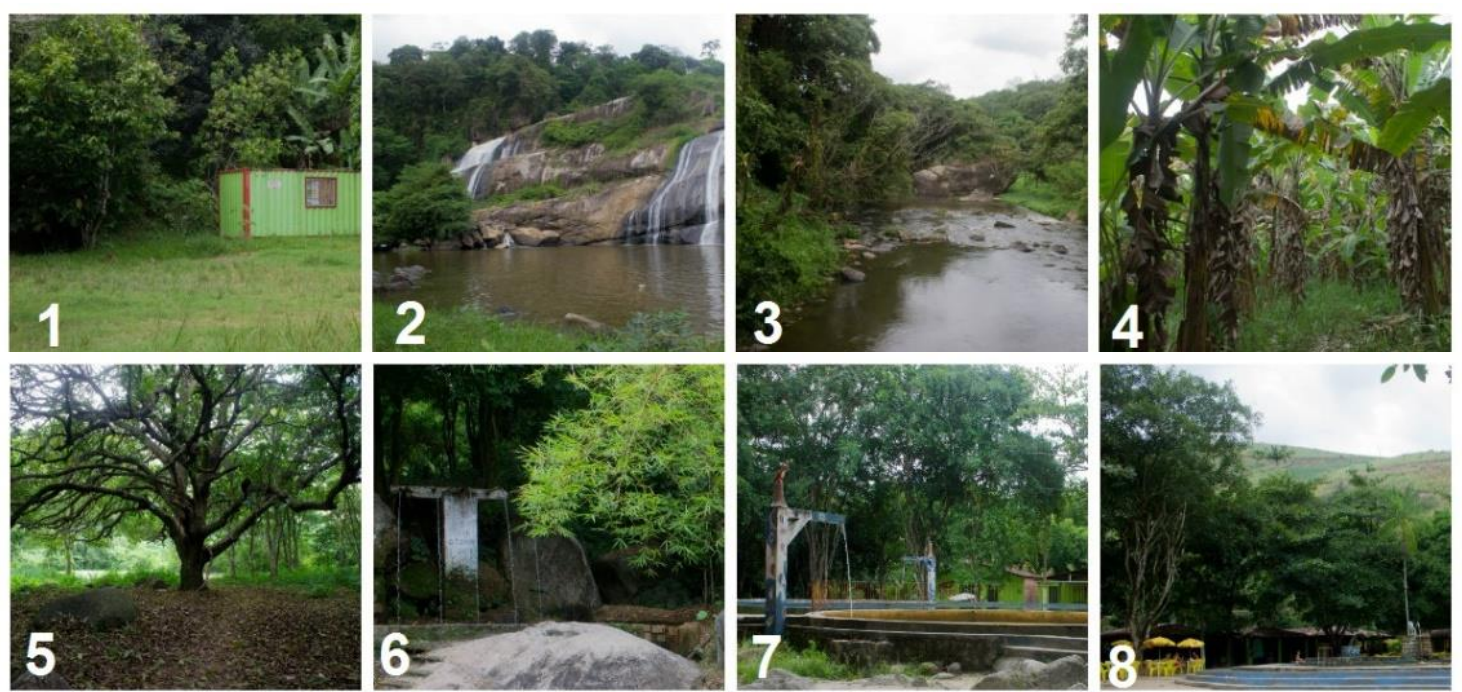

\section{Figura 3}
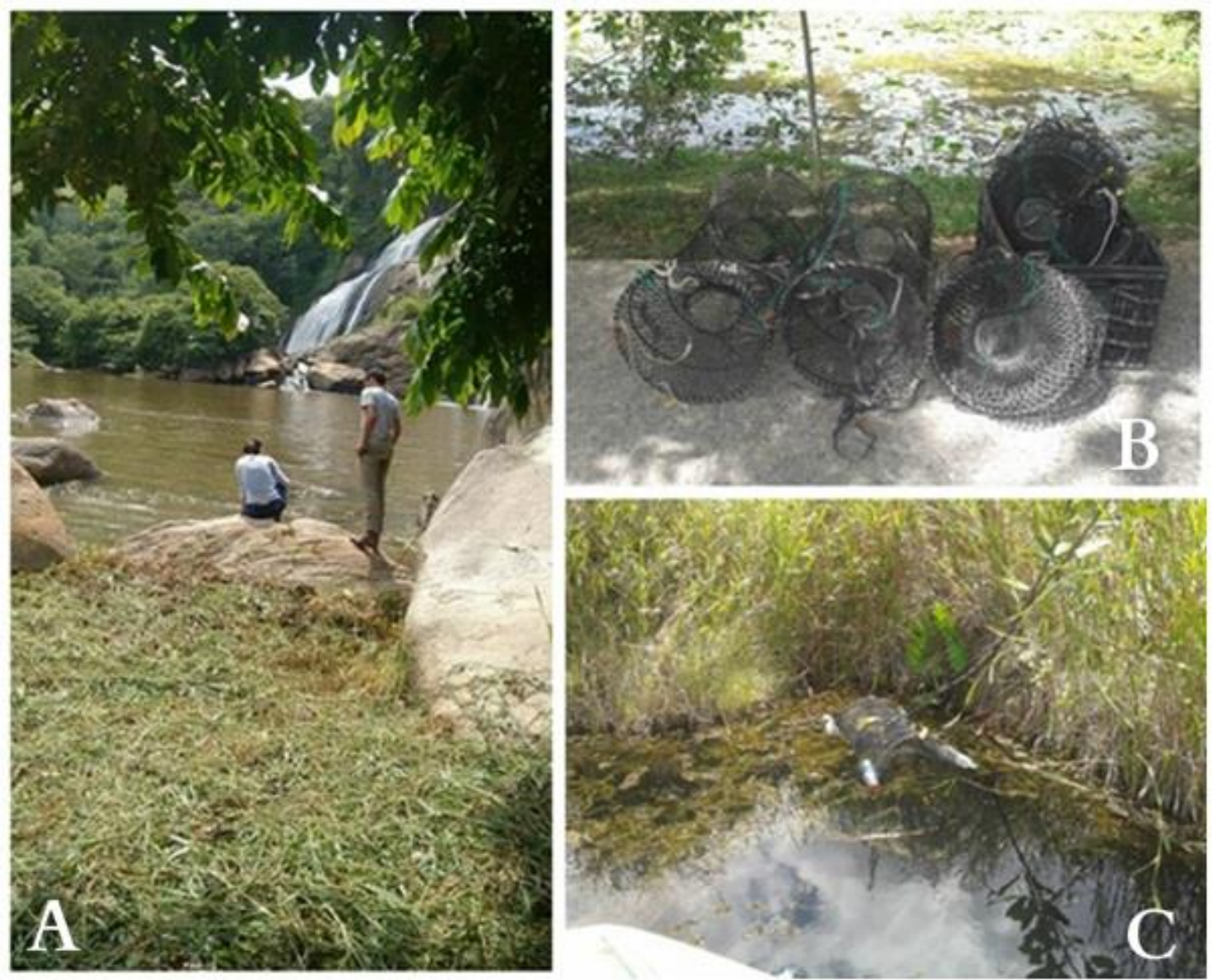
Tabela 2

\begin{tabular}{c|c} 
Táxon & \multicolumn{1}{c}{ Registros } \\
\cline { 2 - 3 } & $\begin{array}{c}\text { Ambiente } \\
\text { de } \\
\text { registro }\end{array}$ \\
\hline
\end{tabular}

\section{Ordem Anura}

\section{Bufonidae}

Rhinella crucifer (Wied-Neuwied, 1821)•

A2(e), Busca

$\mathrm{Ct}$

4

Rhinella jimi (Stevaux, 2002)

A3(g) ativa

A2(e), Busca $\quad \mathrm{Ct}$

A3(g) ativa

\section{Craugastoridae}

Pristimantis ramagii (Boulenger,

A2(d, e) Busca

$\mathrm{Ct}$

1888)

ativa

\section{Hylidae}

Dendropsophus cf. branneri

(Cochran, 1948)

Boana albomarginata (Spix, 1824)

$\begin{array}{llll}\text { A2 }(e, f) & \text { Busca } & \text { Ct } & 28\end{array}$

$\begin{array}{llll}\text { A1(a), } & \text { Busca } & \mathrm{Ct} & 9\end{array}$

A2(e), ativa

$\mathrm{A} 3(\mathrm{~g})$

Boana atlantica (Caramaschi \&

A2(e, f) Busca

As

Velosa, 1996)•

ativa

Boana crepitans (Wied-Neuwied, 1824)

A2 (f) Busca

Ac

1

Boana raniceps (Cope, 1862)

A2 (f) Busca

Ac

1

A1(c), Busca

$\mathrm{Ct}$

Boana semilineata (Spix, 1824) A2(e) (f) ativa

Phyllodytes luteolus (Wied-

Neuwied, 1824)

$\begin{array}{ll}\text { A1(a), } & \text { Busca } \\ \text { A2(f) } & \text { ativa } \\ \text { A3(g) } & \end{array}$

Scinax auratus (Wied-Neuwied, A2(f)

Busca

As

1821)

Scinax nebulosus (Spix, 1824)

A2(e) Busca

As

8

Scinax $x$-signatus (Spix, 1824)

A1(a), Busca

$\mathrm{Ct}$ 


\section{Adenomera marmorata \\ (Steindachner, 1867)}

Leptodactylus fuscus (Schneider, 1799)

Leptodactylus natalensis (A. Lutz, 1930)•

Leptodactylus troglodytes (A. Lutz, 1926)

Leptodactylus vastus(A. Lutz, 1930)

Physalaemus cuvieri (Fitzinger, 1826)•

Pseudopaludicola mystacalis (Cope, 1887)•

\section{Ranidae}

Lithobates palmipes (Spix, 1824)

\section{$\mathrm{A} 2(\mathrm{e}, \mathrm{f})$}

\section{Répteis}

\section{Testudines}

\section{Chelidae}

Phrynops geoffroanus (Schweigger, 1812)

\section{Squamata/Lagartos}

\section{Gekkonidae}

Hemidactylus mabouia (Moreau de Jonnès, 1818)•

\section{Iguanidae}

Iguana iguana (Linnaeus, 1758)

\section{Polychrotidae}

Polychrus marmoratus (Linnaeus, 1758)

\section{Teiidae}

Salvator merianae (Duméril \& Bibron, 1839)
A3(g)

A2(f), Busca

$\mathrm{A} 3(\mathrm{~g}) \quad$ ativa

A2(f) Busca ativa

A2(f), Busca A3(g) ativa

A2(e) Busca

(f), ativa

A3(g)

A1(b), Busca

A2(f)

ativa

A1(b) Busca

ativa

$\begin{array}{cc}\text { A1(b e } & \text { Busca } \\ \text { c) } & \text { ativa }\end{array}$

As

Ac

1

$\mathrm{Ct}$

As

5

$\mathrm{Ct}$

As

5

$\begin{array}{ll}\mathrm{Ct} & 21\end{array}$

Ac

1

A1(c) Busca

As

19

passiva

A1(a, b), Busca $\quad \mathrm{Ct} \quad 20$

A2(f), ativa

A3(g)

A3(g) Relato

Ac

1

terceiros

A2(d) Busca

Ac

1 ativa

A3(g) Relatos

Ac

1 terceiros 
Tropidurus hispidus (Spix, 1825)

Tropidurus semitaeniatus (Spix, 1825)

\section{Squamata/Serpentes}

\section{Boidae}

Boa constrictor (Linnaeus, 1758)

Epicrates cenchria (Linnaeus, 1758)

$\begin{array}{lcll}\text { A3(g) } & \begin{array}{c}\text { Relatos } \\ \text { de } \\ \text { terceiros }\end{array} & \text { Ac } & 1 \\ \text { A2(f) } & \begin{array}{c}\text { Relatos } \\ \text { de } \\ \text { terceiros }\end{array} & \text { Ac } & 1\end{array}$

\section{Colubridae}

Chironius carinatus (Linnaeus, 1758)

Chironius flavolineatus (Jan, 1863)

\section{Dipsadidae}

Clelia plumbea (Wied, 1820)

Erythrolamprus viridis (Günther, 1862)

Oxyrhopus petolarius (Linnaeus, 1758)

Oxyrhopus trigeminus (Duméril, Bibron \& Duméril, 1854)

\section{Elapidae}

Micrurus ibiboboca (Merrem, 1820)

\section{Viperidae}

Crotalus durissus (Linnaeus, 1758)

Lachesis muta (Linnaeus, 1766)
A2(f), Busca

A3(h) ativa

A1(b) Busca Ac

Ac

$\mathrm{A} 3(\mathrm{~g})$ ativa

\section{A1(c) Busca Ac}

Ac

1

A1(a) Busca $\quad$ Ac $\quad 1$

A3(g) Busca $\quad$ Ac $\quad 1$

A1(b) $\quad$ Busca $\quad$ Ac $\quad 1$ ativa

A3(g) Relatos Ac

1 de terceiros

A3(g) Relatos

Ac

1 


\section{Figura 4}

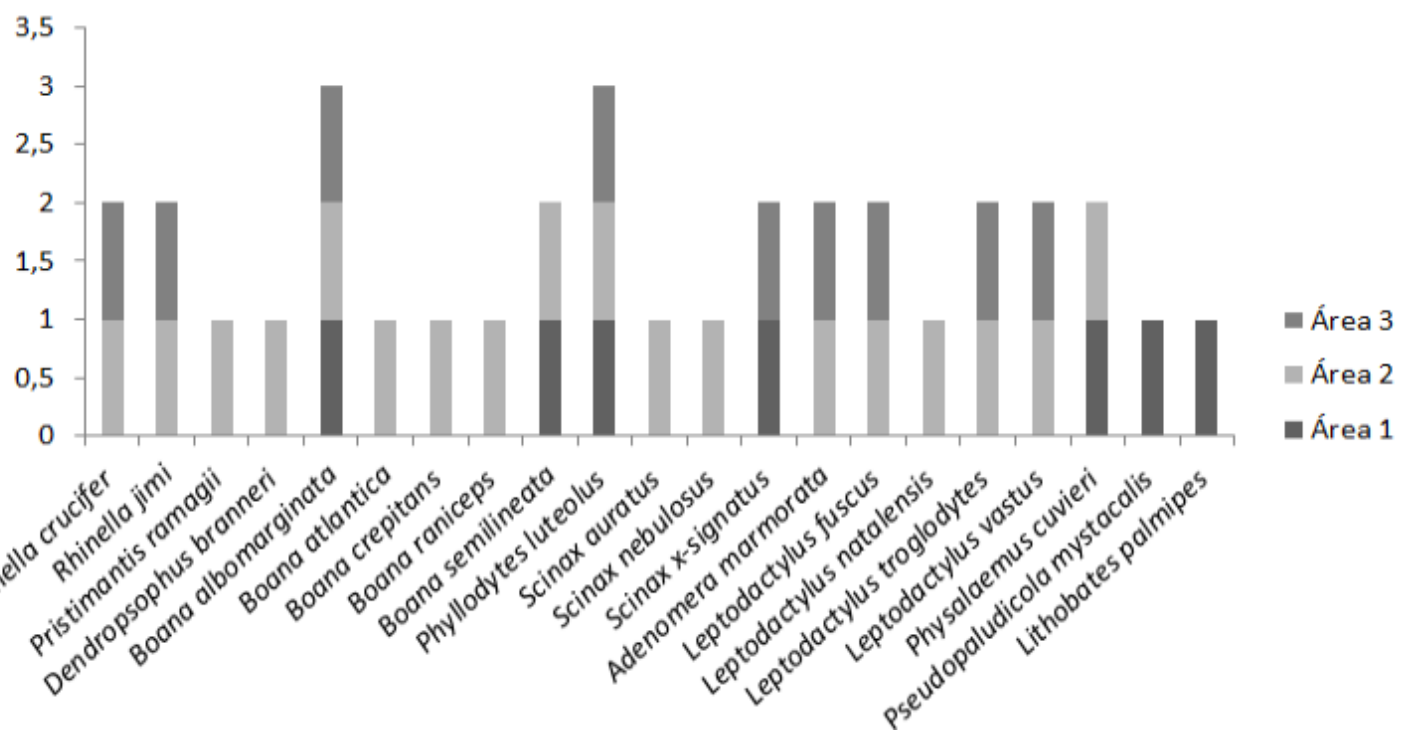

Figura 5
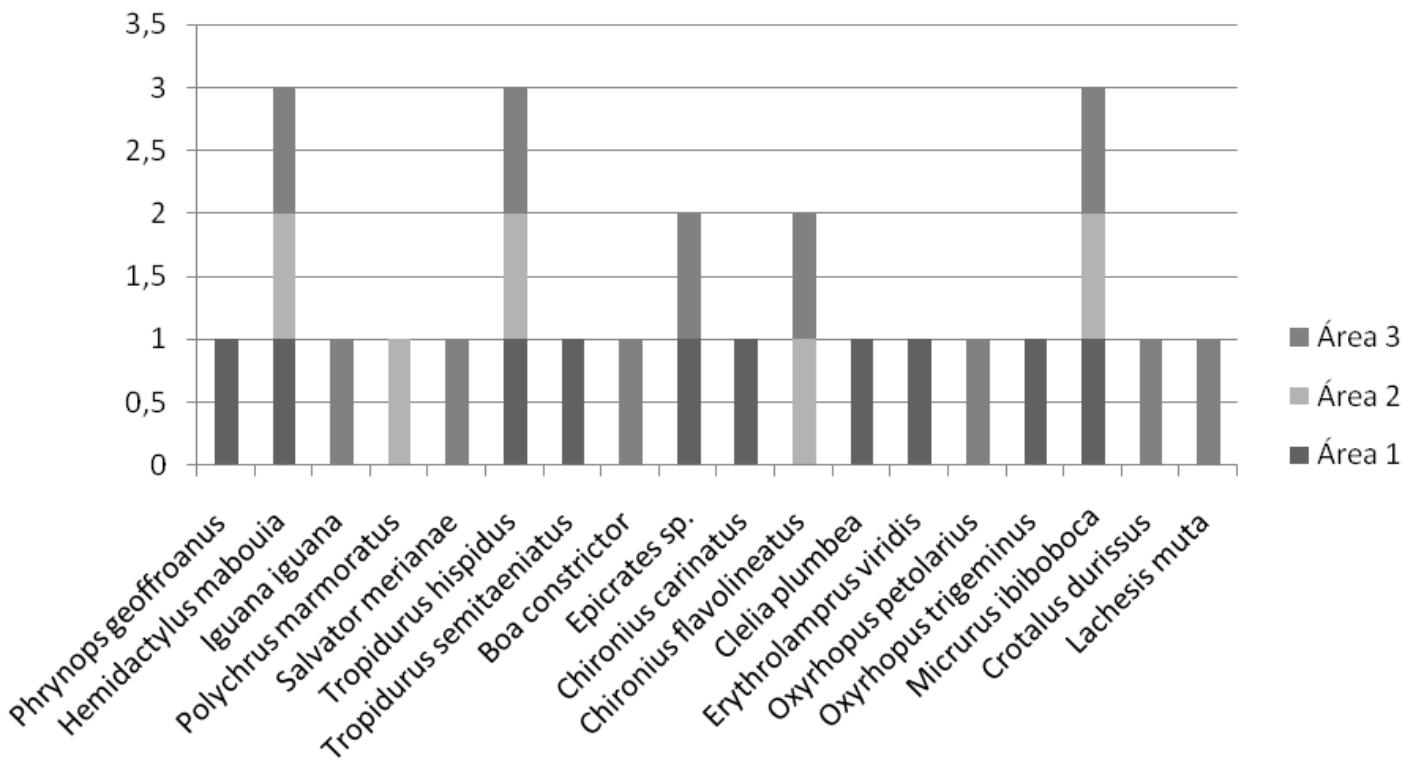

\section{Referências}

1. ALVES, F. Q.; ARGÔLO, A. J. S.; CARVALHO, G. C. Reproductive biology of the bushmaster Lachesis muta (Serpentes: Viperidae) in the Brazilian Atlantic Forest. Phyllomedusa, v. 13, n. 9, p. 99-109, 2014. http://dx.doi.org/10.11606/issn.23169079.v13i2p99-109.

2. ALVES, R. R. N. et al. Répteis e as populações humanas no Brasil: uma abordagem etnoherpetológica. In: ALVES, R. R. N. et al. A Etnozoologia no Brazil - Importância, Status atual e Perspectivas. NUPEEA, 2010, p. 123-147. 
3. ARAUJO, D. F. S.; LUNA, K. P. O. Os répteis e sua representação social: uma abordagem $\begin{array}{lllll}\text { etnozoológica. } \quad \text { Ethnoscientia, } 2017 . & \text { v. }\end{array}$ http://dx.doi.org/10.22276/ethnoscientia.v2i1.61

4. BERNARDE P. S. Anfíbios e Répteis - Introdução ao estudo da herpetofauna brasileira. Anolis Books, 2012, 1 ed.

5. BERNARDE P. S. Serpentes peçonhentas e acidentes ofídicos no Brasil. Anolis Books, 2014, 1 ed.

6. BERTOLUCI, J.; CANELAS, M. A. S.; EISEMBERG, C. C.; PALMUTI, C. F. S. MONTIGELLI, G. C. Herpetofauna da Estação Ambiental de Peti, um fragmento de Mata Atlântica do estado de Minas Gerais, sudeste do Brasil. Biota Neotropica, vol. 9, n. 1, p. 147-155, 2009.

7. CALDAS, F. L. S.; COSTA, T. B.; LARANJEIRAS, D. O.; MESQUITA, D. O.; GARDAN, A.A. Herpetofauna of protected areas in the Caatinga V: Serido Ecological Station (Rio Grande do Norte, Brazil). Check List v. 12, n. 1, p. 1-14, 2016. http://dx.doi.org/10.15560/12.4.1929

8. Dajoz, R. Ecologia geral. Vozes, 1983, 4 ed.

9. COSTA, H.C.; BÉRNILS, R.S. Répteis do Brasil e suas Unidades Federativas: Lista de espécies 2018. Herpetologia Brasileira, v. 7, n. 1, p. 11-57. 2018.

10. FILHO, C. C. A. L. Estrutura da comunidade de lagartos da reserva de Gurjaú, Pernambuco, Brasil. 2003. 92 p. Dissertação (Programa de pós-graduação em Biologia Animal) Universidade Federal de Pernambuco - UFPE, 2003.

11. HADDAD, C. F. B.; PRADO, C. P. A. Reproductive modes in frogs and their unexpected diversity in the Atlantic Forest of Brazil. Bioscience, v. 55, n. 3, p. 207-217, 2005. doi.org/10.1641/0006-3568(2005)055[0207:RMIFAT]2.0.CO;2

12. HADDAD, C. F. B.; TOLEDO, L. F.; PRADO, C. P. A.; LOEBMANN, D.; GASPARINI, J. L.; SAZIMA, I. Guia dos Anfíbios da Mata Atlântica - Diversidade e Biologia. Anolis Books, 2013, 1 ed.

13. LIMA, M. L. C. A Reserva da Biosfera da Mata Atlântica em Pernambuco. RBMA, 1998, 12 ed.

14. LOPES, P. C. Distribuição e abundância de anfíbios e répteis neotropicais em paisagem silvicultural em São Paulo, Brasil. 2010. 78 p. Dissertação (Programa de pós-graduação em ecologia aplicada) - Universidade de São Paulo - USP, 2010.

15. GRANDINETTI, L.; JACOBI, M. C. Distribuição estacional e espacial de uma taxocenose de anuros (Amphibia) em uma área antropizada em Rio Acima - MG. Lundiana v. 6, n. 1, p. 21-28, 2005.

16. GUEDES, T. B.; NOGUEIRA, C.; MARQUES, O. A. V. Diversity, natural history, and geographic distribution of snakes in the Caatinga, Northeastern Brazil. Zootaxa v. 3863, n. 1, p. 001-093, 2014. http://10.11646 / zootaxa.3863.1.1

17. MELGAREJO, A. R. Criando serpentes salvando vidas. Rio Book's, 2013, 1 ed.

18. MELLO, K.; TOPPPA, R. H.; CARDOSO-LEITE, E. Priority areas for forest conservation in an urban landscape at the transition between Atlantic Forest and Cerrado. CERNE, v. 22, n. 3, p. 277-288, 2016. http://dx.doi.org/10.1590/01047760201622032172.

19. MOTTA-TAVARES, M.; MAIA-CARNEIRO, T.; DANTAS, L. F.; SLUYS, M. V.; HATANO, F. H.; VRCIBRADIC, D.; ROCHA, C. F. D. Ecology of the bromeligenous frog Phyllodytes luteolus (Anura, Hylidae) from three restinga remnants across Brazil's coast. Anais da Academia Brasileira de Ciências, v. 88, n. 1, p. 93-104, 2016. http://dx.doi.org/10.1590/0001-3765201620140380

20. MOURA, G. J. B. et al. Distribuição Geográfica e Caracterização Ecológicas dos Répteis do Estado de Pernambuco. In: Moura, G. J. B. et al. Herpetologia do Estado de Pernambuco. Ministério do Meio Ambiente/IBAMA, 2011a, p. 229-290. 
21. MOURA, G. J. B. et al. Distribuição geográfica e caracterização ecológica dos anfíbios de Pernambuco. In: Moura, G. J. B. et al. Herpetologia no estado de Pernambuco. Ministério do Meio Ambiente/IBAMA, 2011b, p. 51-84.

22. MURIARTY, J. J. Reptiles as pets: an examination of the trade in live reptiles in the United States. Herpetological Review, v. 33, n. 3, 2002.

23. OLIVEIRA, O. P. Avaliação da composição química e atividade antimicrobiana e antiinflamatória do óleo extraído da gordura corporal de Spilotes pullatus (Linnaeus, 1758) (Colubridae: Ophidia) da Chapada do Araripe no Nordeste brasileiro. 2013. 72 p. Dissertação (Programa de Pós-Graduação em Bioprospecção Molecular) Universidade Regional do Cariri - URC, 2013.

24. PETERS, J. A.; OREJAS-MIRANDA, B. Catalogue of the Neotropical Squamata. Part I. Snakes. United States National Museum Bulletin, v. 297, p. 1-347, 1970.

25. PETERS, J. A.; BARROS, D. Catalogue of the Neotropical squamata. Part II. Lizards and Amphisbaenians. United States National Museum Bulletin, v. 297, p. 1-293, 1970.

26. PEREIRA, E. N.; LIRA, C. S.; SANTOS, E. M. Ocupação, distribuição espacial e sazonal dos anfíbios anuros, em fragmento de mata atlântica. Revista Ibero-Americana de Ciências Ambientais, v. 7, n. 2, p. 70-83, 2016. https://doi.org/10.6008/SPC21796858.2016.002.0006

27. ROBERTO, I. J.; OLIVEIRA, C. R.; FILHO, J. A. A.; OLIVEIRA, H. F.; ÁVILA, R. W. The herpetofauna of the Serra do Urubu mountain range: a key biodiversity area for conservation in the brazilian Atlantic Forest. Papéis Avulsos de Zoologia, v. 57, n. 27, p. 343-373, 2017. http://dx.doi.org/10.11606/0031-1049.2017.57.27

28. RODRIGUES, M. T. The conservation of Brazilian reptiles: challenges of a megadiverse country. Conservation Biology, v. 19, n. 3, p. 659-664, 2005.

29. SANTANA, G. G.; VIEIRA, W. L. S.; PEREIRA-FILHO, G. A.; DELFIM, F. R.; LIMA, Y. C. C.; VIEIRA, K. S. Herpetofauna em um fragmento de Floresta Atlântica no Estado da Paraíba, Região Nordeste do Brasil. Biotemas, v. 21, n. 1, p. 75-84, 2008. https://doi.org/10.5007/2175-7925.2008v21n1p75

30. SANTANA, D. O.; CALDAS, F. L. S.; GOMES, F. F. A.; SANTOS, R. A.; SILVA, B. D.; ROCHA, S. M.; FARIA, R. G. Natural History aspects of Tropidurus hispidus in na area of Atlantic Forest, northeastern Brazil. Neotropical Biology and Conservation, v. 9, n. 1, p. 55-61, 2014. https://10.4013/nbc.2014.91.07

31. SANTOS, A. M. M. Flora do Centro de Endemismo Pernambuco: Biogeografia e Conservação 2006. 160 p. Dissertação (Programa de Pós-Graduação em Biologia Vegetal) - Universidade Federal de Pernambuco - UFPE, 2006.

32. SANTOS, N. E. F.; DIAS, U. N. S.; BEZERRA, J. K.; SILVA, C. D. A.; RIBEIRO, L. B. Frugivoria e dispersão de sementes por lagartos em ecossistemas brasileiros: uma revisão. Revista Nordestina de Zoologia, v. 6, n. 2, p. 74-102, 2012.

33. SANTOS, S.P. L.; SANTOS, E.M. Anurofauna da Reserva Particular do Patrimônio Natural Frei Caneca, município de Jaqueira, estado de Pernambuco, Brasil. In: Moura, G.J.B. et al. Herpetologia no estado de Pernambuco. IBAMA, 2011, p. 187-198.

34. SEGALLA, M. V.; CARAMASCHI, U.; CRUZ, C. A. G.; GRANT, T.; HADDAD, C. F. B.; GARCIA, P. C. A.; BERNCEK, B. V. M.; LANGONE, J. A. Brazilian Amphibians: List of Species. Herpetologia Brasileira, v. 5, p. 34-46, 2016.

35. SOUZA, K.; COELHO, R. D. F.; SOBRINHO, C. P.; NASCIMENTO, J. P. B.; GOGLIATH, M.; RIBEIRO, L. B. Fauna de lagartos de habitats de Caatinga do Campus Ciências Agrárias da Universidade Federal Do Vale Do São Francisco, Petrolina-Pe, Brasil. Revista Brasileira de Zoociências,v. 15, p. 253-265, 2013.

36. UCHOA-NETO, C. A. M. U.; TABARELLI, M. Diagnóstico e Estratégia de Conservação do Centro de Endemismo Pernambuco Centro de Pesquisas Ambientais do Nordeste CEPAN Termo de Referência $N^{\circ}$ CS FY02 / 00X Conservation International do Brasil Recife, Julho. 2002. 